\title{
EDITORIAL \\ Deep brain stimulation for refractory posttraumatic stress disorder
}

\author{
Justin D. Hilliard, MD, ${ }^{1}$ and Kelly D. Foote, MD ${ }^{1,2}$ \\ 1Department of Neurosurgery, and ${ }^{2}$ Fixel Center for Neurological Disorders, University of Florida, Gainesville, Florida
}

$\mathrm{P}$ OSTTRAUMATIC stress disorder (PTSD) is an anxiety disorder resulting in functional impairment as a result of exposure to threatened or actual death, serious injury, or sexual violence. The traumatic event is persistently re-experienced with intrusions of thoughts, feelings, or reminders, with associated excessive emotional arousal including hypervigilance, difficulty concentrating, and irritability. Individuals affected with the disorder often socially isolate themselves to avoid trauma-related stimuli, and frequently have concomitant depression. Historically, PTSD treatments have consisted of cognitive behavioral therapy, exposure therapy, and pharmacological treatment with SSRIs (selective serotonin reuptake inhibitors) and SNRIs (serotonin-norepinephrine reuptake inhibitors). Lifetime prevalence of the disorder is as high as $7 \%$ in the United States. ${ }^{1}$ Combat veterans are classically at high risk for PTSD, with a $15 \%$ prevalence in male Vietnamera veterans. ${ }^{10}$ Although significant advances have been made in the cognitive and pharmaceutical therapies for PTSD, in a subset of the population the disorder remains refractory to treatment. Refractory PTSD is probably partially due to lack of fear extinction, rendering exposure therapy ineffective. ${ }^{11}$ Fear extinction is modulated by the basolateral nucleus (BLn) of the amygdala and the medial prefrontal cortex, the former of which demonstrates amplified blood flow and metabolism on functional imaging in refractory PTSD. ${ }^{4,5}$ This poses a unique treatment challenge for which electrical neuromodulation may be particularly well suited. ${ }^{12}$

The BLn of the amygdala is hypothesized to be an effective target for deep brain stimulation (DBS) for PTSD because it serves as a mediator for emotional and autonomic responses to stimuli in the environment. The BLn is a relay station within the amygdala, connecting the lateral nucleus with the central nucleus and ultimately forming a feedback loop with the medial prefrontal cortex.? Extinction cells and fear-encoding cells are located within the BLn. When a sensory stimulus is applied, the relative activity of "fear" cells to "extinction" cells determines the individual's ultimate behavioral response..$^{10}$ Modulating the BLn to enhance extinction while mitigating fear could reduce the emotional arousal associated with stimuli conditioned to the index traumatic event. Functional imaging studies have demonstrated that individuals with PTSD have hyperactivity in the amygdala, particularly within the basal portion, compared to controls. ${ }^{5}$ The intensity of such hyperactivity appears linked to the magnitude of symptoms. ${ }^{2}$ It is hypothesized that as with other psychiatric conditions characterized by hyperactive networks, such as depression and obsessive-compulsive disorder, DBS applied to the hyperactive amygdala could moderate function in connected regions, thereby suppressing symptoms of PTSD.

More refined neuromodulation with closed-loop DBS could theoretically be used in a PTSD application, with biomarkers reflecting sympathetic activation (skin conductance changes, pupillary dilation, piloerection). However, these peripheral cues inherently lack specificity, because normal emotional responses could trigger activation. Characteristic neuronal activity (e.g., local field potentials) from the amygdala or medial prefrontal cortex itself may be a more specific and more easily integrated biomarker into a closed-loop DBS system. ${ }^{3}$

In their paper titled "Bilateral amygdala stimulation reduces avoidance behavior in a predator scent posttraumatic stress disorder model," Dengler et al. outline their trial of bilateral basolateral amygdala DBS in a rodent PTSD model. ${ }^{6}$ Previous DBS studies in animals with PTSD have used an aversive conditioning model whereby a foot shock stimulus is paired with an object placed in the environmental chamber at the same time as the shock. The rats, when exposed again to the innocuous object, will bury it in an attempt to avoid the shock stimulus. The study's authors argue that the predator scent model, in which the 
rodent is exposed to a life-threatening, stress-inducing stimulus without direct physical repercussion, is perhaps more in line with the types of cues that humans encounter in a situation leading to PTSD. The scent model produces prolonged anxiety and avoidance behaviors, in contrast to the relatively short-acting effects of the aforementioned aversive conditioning model.

In the aforementioned study, rats were assigned to one of four conditions: bilateral stimulation, unilateral stimulation, no stimulation, and no stimulation/no exposure to the predator scent. Stimulation was applied for 4 hours a day for 1 week prior to animals undergoing repeat behavioral testing. Electrode placement was validated with postmortem histological investigation. The study demonstrated that bilateral high-frequency stimulation resulted in decreased avoidance behavior compared to the no-stimulation cohort. Interestingly, the stimulation cohort did not demonstrate decreased anxiety, as measured by the lack of a significant change in time spent on the open arm portion of the elevated plus maze. One shortcoming of the authors' study is the lack of control to negate the potential influence of a lesion effect. A separate condition of predator scent exposure without implantation should be tested. In human studies, damage to the amygdala has been shown to have a protective effect from PTSD, thereby highlighting the importance of ensuring that the effects noted in the study are not in part from implantation alone.

As DBS indications continue to mature beyond movement disorders into the realm of psychiatric and cognitive disorders, the need for careful selection of anatomical targets, development of stringent implant criteria, and a consensus of ethical considerations will become critically important. Well-designed preclinical studies are helpful in narrowing the vast array of plausible anatomical targets. Given the promising results, already there are groups that have extended DBS for PTSD beyond animal models. Langevin et al. implanted bilateral BLn DBS electrodes in a 48-year-old combat veteran with severe, refractory PTSD. ${ }^{11}$ After 8 months of continuous stimulation, he had experienced a $38 \%$ decrease in his clinician-administered PTSD (CAPS) score, which is a measure of PTSD severity. The results from this initial subject led to the launch of a Phase I pilot study investigating bilateral BLn DBS for 6 combat veterans with refractory PTSD. ${ }^{8,9}$ For those with refractory PTSD, DBS may offer not only a life-modifying therapy, but in fact a life-saving one.

https://thejns.org/doi/abs/10.3171/2018.5.FOCUS18266

\section{References}

1. American Psychiatric Association: Diagnostic and Statistical Manual of Mental Disorders, ed 5. Washington, DC: American Psychiatric Association, 2013
2. Armony JL, Corbo V, Clément MH, Brunet A: Amygdala response in patients with acute PTSD to masked and unmasked emotional facial expressions. Am J Psychiatry 162:19611963,2005

3. Bina RW, Langevin JP: Closed loop deep brain stimulation for PTSD, addiction, and disorders of affective facial interpretation: review and discussion of potential biomarkers and stimulation paradigms. Front Neurosci 12:300, 2018

4. Bryant R, Felmingham K, Kemp A: Amygdala and ventral anterior cingulate activation predicts treatment response to cognitive behaviour therapy for post-traumatic stress disorder. Psychol Med 38:555-561, 2008

5. Bryant RA, Felmingham KL, Kemp AH: Neural networks of information processing in posttraumatic stress disorder: a functional magnetic resonance imaging study. Biolog Psychiatry 58:111-118, 2005

6. Dengler BA, Hawksworth SA, Berardo L, McDougall I, Papanastassiou AM: Bilateral amygdala stimulation reduces avoidance behavior in a predator scent posttraumatic stress disorder model. Neurosurg Focus 45(2):E16, 2018

7. Freese JL, Amaral DG: Neuroanatomy of the primate amygdala, in Whalen PJ, Phelps EA (eds): The Human Amygdala. New York: Guilford Press, 2009

8. Koek RJ, Langevin JP, Krahl SE: Deep brain stimulation of the basolateral amygdala for treatment-refractory combat post-traumatic stress disorder (PTSD): study protocol for a pilot randomized controlled trial with blinded, staggered onset of stimulation. Trials 15:356, 2014

9. Langevin JP, Chen JW, Koek RJ: Deep brain stimulation of the basolateral amygdala: targeting technique and electrodiagnostic findings. Brain Sci 6:E28, 2016

10. Langevin JP, Koek RJ, Schwartz HN, Chen JWY, Sultzer DL, Mandelkern MA, et al: Deep brain stimulation of the basolateral amygdala for treatment-refractory posttraumatic stress disorder. Biol Psychiatry 79:e82-e84, 2016

11. Laufer RS, Brett E, Gallops MS: Dimensions of posttraumatic stress disorder among Vietnam veterans. J Nerv Ment Dis 173:538-545, 1985

12. Marin MF, Camprodon JA, Dougherty DD, Milad MR: Device-based brain stimulation to augment fear extinction: implications for PTSD treatment and beyond. Depress Anxiety 31:269-278, 2014

\section{Disclosures}

Dr. Foote received support from Medtronic, Abbott/St. Jude, Boston Scientific, and Functional Neuromodulation for nonstudy-related clinical or research efforts that he oversaw. He is also a patent holder for DBS-related inventions, and receives fellowship grant support from Medtronic.

\section{Correspondence}

Kelly D. Foote: foote@neurosurgery.ufl.edu.

\section{INCLUDE WHEN CITING}

DOI: $10.3171 / 2018.5 . F O C U S 18266$. 